\title{
Tabaquismo e Infarto agudo al Miocardio
}

\author{
MAURICIO FERNÁNDEZ R.*
}

\section{Smoking and acute myocardial infarction}

The most important measure to prevent a new acute coronary event in a smoking patient is cessation of smoking. To achieve this goal, brief counseling should be conducted according to the Guidelines for Clinical Practice of Smoking Treatment, Chile 2017 in the hospitalized patient as soon as possible, and the cessation of the addiction must be extended post-discharge by referral to a center where intensive smoking cessation is performed.

Key words: Tobacco use; myocardial infarction; smoking cessation; smoking; referral and consultation.

\section{Resumen}

La medida más importante para prevenir un nuevo evento coronario agudo en un paciente fumador es la cesación del tabaquismo. Para conseguir esta meta, se debe realizar consejería breve según las Guías de Práctica Clínica de Tratamiento del Tabaquismo, Chile 2017 en el paciente hospitalizado lo antes posible, y la ayuda en cesación debe extenderse posterior al alta derivando a un centro donde se realice tratamiento intensivo de cesación del tabaquismo.

Palabras clave: Tabaquismo; infarto del miocardio; fumar; dejar de fumar; derivación y consulta.

Las enfermedades relacionadas con el consumo de tabaco son una de las principales causas de ingresos hospitalarios, de éstas, las enfermedades cardiovasculares están dentro de las más importantes.

Un $25 \%$ de todas las muertes evitables entre los fumadores, son causadas por enfermedades cardiovasculares. Las personas expuestas al humo de segunda mano tienen un aumento de 20 a 30 por ciento en el riesgo de morbilidad y mortalidad causada por una enfermedad coronaria ${ }^{1}$.

Posterior a un evento cardiovascular como un Infarto agudo al miocardio con o sin supradesnivel ST en el ECG, un cuadro de angina inestable o un procedimiento de revascularización coronaria, se deben considerar una serie de medidas de prevención secundaria.

Dentro de éstas, se plantea como meta la completa cesación del hábito tabáquico. Tanto las guías americanas, como las europeas de manejo de síndrome coronario agudo recomiendan: eva- luar el uso de tabaco, recomendar fuertemente al paciente y familiares el dejar de fumar y a evitar el humo de segunda mano. Entregar consejería, terapia farmacológica e ingresar a un programa de cesación de tabaco².

Varios meta-análisis en pacientes posterior a un síndrome coronario agudo han mostrado que la cesación tabáquica reduce en aproximadamente un $50 \%$ mortalidad futura en este grupo de pacientes, constituyéndose en una de las medidas más poderosas de prevención secundaria ${ }^{3,4}$.

La hospitalización de un paciente fumador, es un evento que constituye una gran oportunidad para aplicar medidas de cesación de tabaquismo. Es un momento en que el paciente se replantea su estilo de vida y está abierto a cambios hacia hábitos más saludables.

Dentro de las estrategias a seguir durante la hospitalización están, la $\mathrm{ABC}-\mathrm{D}$, el ingreso a un programa de rehabilitación cardiaca y el uso de terapia farmacológica para la cesación del taba-

* Dpto. Cardiología, Clínica Alemana de Santiago.

Presidente, Departamento de Prevención, Sociedad Chilena de Cardiología. 
quismo si el paciente es adicto y quiere dejar de fumar.

Respecto a la consejería, esta debe implementarse desde el momento en que el paciente es hospitalizado, preguntando por el consumo de tabaco, aconsejando sobre su abandono y los beneficios de que esto conlleva. Mientras más profesionales estén involucrados en dar consejo (médicos, enfermeras, kinesiólogos, auxiliares, otros), se ha demostrado que éste es más efectivo. Así también, el nivel de intensidad de la consejería lleva a mejores resultados. En la revisión de Rigotti y cols., en Cochrane, una intervención hospitalaria de más de 15 min asociada a un seguimiento al alta mas allá del primer mes, fue el más eficiente ${ }^{6}$.

Respecto a la terapia farmacológica, se acepta el uso de terapia de reemplazo nicotínico (TRN), el uso de bupropión y vareniclina.

Si bien estos tres fármacos son aceptados como alternativas terapéuticas, en el paciente hospitalizado por un infarto agudo al miocardio, no queda clara la efectividad de bupropión, por lo que no está recomendado su uso en este escenario ${ }^{4,8}$.

En cuanto a TRN, si bien no hay estudios randomizados controlados, se recomienda su uso desde que se ha estabilizado al paciente (hemodinamia estable, ausencia de arritmias graves). Se puede usar en parches, chicles o spray bucal. $\mathrm{Su}$ uso ha demostrado ser seguro ${ }^{4,6,7}$.

Recientemente el estudio EVITA ${ }^{5}$ evaluó el uso de vareniclina en paciente fumadores hospitalizados por síndrome coronario agudo. El estudio mostró una tasa significativamente mayor de abandono del tabaco a los 6 meses post infarto en el grupo con vareniclina en relación con placebo (47\% vs 32\%), sin diferencias en complicaciones cardiovasculares.

El abandono del tabaco debe ser una prioridad para la prevención primaria y secundaria de la enfermedad coronaria y el tabaquismo debe ser tratado de la misma forma que otros factores de riesgo importantes como diabetes, hipertensión y dislipidemia. La cesación del tabaquismo implica una mayor reducción de la mortalidad por enfermedad cardiovascular que cualquier otra intervención de prevención secundaria incluyendo el uso de $\beta$-bloqueadores, inhibidores de la enzima convertidora de angiotensina, estatinas o aspirina. $Y$, por último, los beneficios de los fármacos antihipertensivos o hipolipemiantes se reducen significativamente en los pacientes que no dejan de fumar ${ }^{1}$.

Como conclusión es importante señalar que lograr la cesación del consumo de tabaco posterior a un síndrome coronario agudo, como ya se ha mencionado, es la medida más efectiva para prevenir un nuevo evento futuro. Para conseguir esta meta, se deben considerar todas las medidas terapéuticas, farmacológicas y no farmacológicas antes mencionadas. Estas deben iniciarse lo más tempranamente posible durante la hospitalización, debe involucrar a todo el personal, médico y de apoyo, a cargo del paciente, y debe extenderse posterior al alta en un programa de rehabilitación cardíaca con duración no menor a un mes posterior al evento.

\section{Bibliografía}

1.- TOB-g. Tobacco Cessation Guidelines for High Risk Population.Disponible en: http://www.tri.ie/ uploads/5/2/7/3/52736649/tob-g-book-digitalversion.pdf (consultado en agosto 2017).

2.- ANTMAN EM, ANBE DT, ARMSTRONG PW, BATES ER, GREEN LA, HAND M, et al. ACC/AHA guidelines for the management of patients with STelevation myocardial infarction-executive summary: a report of the American College of Cardiology/American Heart Association Task Force on Practice Guidelines. Circulation 2004; 110: 588-636.

3.- WILSON K, GIBSON N, WILLAN A, COOK D. Effect of smoking cessation on mortality after myocardial infarction: meta-analysis of cohort studies. Arch Intern Med 2000; 160: 939-44.

4.- THOMSON CC, RIGOTTI NA. Hospital- and clinicbased smoking cessation interventions for smokers with cardiovascular disease. Prog Cardiovasc Dis 2003; 45: 459-79.

5.- EISENBERG MJ, WINDLE SB, ROY N, OLD W, GRONDIN FR, BATA I, et al. EVITA Investigators. Varenicline for Smoking Cessation in Hospitalized Patients With Acute Coronary Syndrome. Circulation 2016; 133: 21-30.

6.- RIGOTTI NA, CLAIR C, MUNAFÒ MR, STEAD LF. Interventions for smoking cessation in hospitalised patients. Cochrane Database of Systematic Reviews 2012, Issue 5. Art. No.: CD001837. DOI: 10.1002/14651858.CD001837.pub3.

7.- JIMÉNEZ RUIZ CA, DE GRANDA ORIVE JI, SOLANO REINA S, RIESCO MIRANDA JA, DE HIGES MARTÍNEZ E, PASCUAL LLEDÓ JF, et al. Guidelines for the Treatment of Smoking in Hospitalized Patients Arch Bronconeumol. 2017 ;53:387-94. doi: 10.1016/j.arbres.2016.11.004

8.- SIMON JA, DUNCAN C, HUGGINS J, SOLKOWITZ S, CARMODY TP. Sustained-release bupropion for hospital-based smoking cessation: a randomized trial. Nicotine Tob Res 2009; 11: 663-9.

Correspondencia a:

Dr. Mauricio Fernández R.

Email: romagnoli.fernandez.m@gmail.com 